\title{
The Lyapunov-Malkin Theorem and Stabilization of the Unicycle with Rider
}

\author{
Dmitry V. Zenkov* \\ Department of Mathematics \\ North Carolina State University \\ Raleigh, NC 27695 \\ dvzenkov@unity.ncsu.edu
}

\author{
Anthony M. Bloch ${ }^{\dagger}$ \\ Department of Mathematics \\ University of Michigan \\ Ann Arbor, MI 48109 \\ abloch@math.lsa.umich.edu
}

\author{
Jerrold E. Marsden ${ }^{\ddagger}$ \\ Control and Dynamical Systems \\ California Institute of Technology 107-81 \\ Pasadena, CA 91125 \\ marsden@cds.caltech.edu
}

March, 2001

Systems and Control Lett. 46, (2002), 293-300.

\begin{abstract}
This paper analyzes stabilization of a nonholonomic system consisting of a unicycle with rider. It is shown that one can achieve stability of slow steady vertical motions by imposing a feedback control force on the rider's limb.
\end{abstract}

\section{Keywords}

Nonholonomic system, underactuated feedback control

\footnotetext{
*Research partially supported by NSF grants DMS-9803181 and DMS-0103895, AFOSR grant F49620-96-1-0100, a University of Michigan Rackham Fellowship, and an NSF group infrastructure grant at the University of Michigan

${ }^{\dagger}$ Research partially supported by NSF grants DMS-9803181 and DMS-0103895, AFOSR grant F49620-96-1-0100, and an NSF group infrastructure grant at the University of Michigan

${ }^{\ddagger}$ Research partially supported by AFOSR grant F49620-95-1-0419
} 


\section{Introduction}

Problem Setting. In this paper we study the stabilization problem for a simplified model of a rider on a unicycle using techniques from the theory of stability of nonholonomic dynamical systems that are given in Zenkov, Bloch, and Marsden [1998]. In the current paper, we make use of the Lyapunov-Malkin theorem, which is recalled below. We incorporate in our analysis the special structure of nonholonomic mechanical systems with symmetry, namely the geometry of dividing the system variables into internal (or shape) variables and momentum variables corresponding to symmetry directions. However, unlike holonomic systems, symmetries need not lead via Noether's theorem to conservation laws; rather, the momenta corresponding to symmetries obey dynamic momentum equations (see Bloch, Krishnaprasad, Marsden, and Murray [1996]). In some cases, Zenkov, Bloch, and Marsden [1998] were able to show that stability of motion could nonetheless be analyzed using a generalization of energy-momentum methods (see e.g. Marsden [1992]). In other cases a combination of spectral and nonlinear analysis was used with the assistance of the Lyapunov-Malkin theorem, which may be viewed as a variant of stability analysis based on the center manifold theory.

Unicycle Model and Methodology. We model the rider on a unicycle in this paper by a pendulum suspended on a rod attached to the center of a wheel, the rod and the pendulum representing the body and the limb of the rider. This leads to complicated but tractable equations. We then apply a linear feedback control to the pendulum representing the limb of the rider, and conclude nonlinear stability of the reduced system using the Lyapunov-Malkin theorem. The Lyapunov-Malkin theorem, which enables one to conclude overall nonlinear stability using partial spectral information about the system, has been particularly useful for the analysis of nonholonomic systems (see Karapetyan [1981], Markeev [1992], and Zenkov, Bloch, and Marsden [1998]). In particular, here we apply this technique to achieve stabilization of slow vertical steady state motions of a homogeneous disk on a horizontal plane with a hanging pendulum attached. Fast motions may also be stabilized and are in fact easier to handle because of the stabilizing effect of the wheel velocity. A brief announcement of some of the ideas discussed here may be found in Zenkov, Bloch, and Marsden [1999], where we stated the results proved in the current paper. In addition, here we present a simulation of our results and discuss the qualitative behavior of the closed loop system. While the analysis in the present work is somewhat involved, 
we intend to extend it both to more complex nonholonomic/robotic systems and to more complicated nonlinear control techniques, for example the matching control technique discussed in Bloch, Leonard, and Marsden [1997, 1998]. In particular, the latter technique allows one to achieve a large basin of attraction while the results here achieve, at least analytically, only local stabilization.

\section{Modeling the Unicycle with Rider}

We now present the dynamical model of a homogeneous disk on a horizontal plane with a mass and pendulum attached. The pendulum is free to move in the plane orthogonal to the disk, while the attached mass stays in the disk's plane. We view this as a simplified model of a rider on a unicycle in which only the sideways motion of the rider (such as the rider's limbs) is modeled, without pedaling control.

Configuration Space. The configuration space for the unicycle with rider as described above is $Q=S^{1} \times S^{1} \times S^{1} \times S E(2)$, which we parameterize with coordinates $(\theta, \varkappa, \psi, \phi, x, y)$. As in Figure $1, \theta$ is the tilt of the unicycle itself, $\varkappa$ is that of the limb, and $\psi$ is the angular position of the wheel of the unicycle. The variables $(\phi, x, y)$, regarded as a point in $S E(2)$, represent the angular orientation and position of the point of contact of the wheel with the ground.

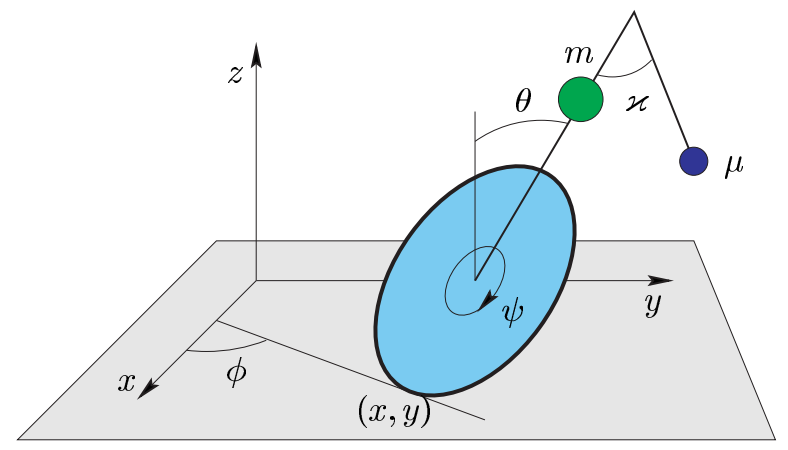

Figure 1: The configuration variables for the unicycle with rider.

The Symmetry Group. This mechanical system is $S O(2) \times S E(2)$ invariant; the group $S O(2)$ represents the symmetry of the wheel, that is, 
the symmetry in the $\psi$ variable, while the group $S E(2)$ represents the Euclidean symmetry of the overall system. The action by the group element $(\alpha, \beta, a, b)$ on the configuration space is given by

$$
\begin{aligned}
& (\theta, \varkappa, \psi, \phi, x, y) \mapsto \\
& \quad(\theta, \varkappa, \psi+\alpha, \phi+\beta, x \cos \beta-y \sin \beta+a, x \sin \beta+y \cos \beta+b) .
\end{aligned}
$$

System Parameters. We will use the following notations:

$$
\begin{aligned}
M & =\text { the mass of the disk, } \\
R & =\text { the radius of the disk, } \\
A, B & =\text { the principal moments of inertia of the disk, } \\
m & =\text { the rider mass, } \\
r & =\text { the rod length, } \\
l & =\text { the distance from the center of the disk to the mass } m, \\
\mu & =\text { the limb mass } \\
\rho & =\text { the limb length. }
\end{aligned}
$$

Lagrangian. The Lagrangian of this system has the standard form of kinetic minus potential energy:

$$
L=K_{\text {disk }}+\frac{m}{2} v_{m}^{2}+\frac{\mu}{2} v_{\mu}^{2}-U,
$$

where,

$$
\begin{aligned}
K_{\text {disk }}= & \frac{1}{2}\left[A\left(\dot{\theta}^{2}+\dot{\phi}^{2} \cos ^{2} \theta\right)+B(\dot{\phi} \sin \theta+\dot{\psi})^{2}\right] \\
& +\frac{M}{2}\left[R^{2} \dot{\theta}^{2}+2 R(\dot{y} \cos \phi-\dot{x} \sin \phi) \dot{\theta} \cos \theta\right. \\
& \left.+(\dot{x}-R \dot{\phi} \sin \theta \cos \phi)^{2}+(\dot{y}-R \dot{\phi} \sin \theta \sin \phi)^{2}\right], \\
v_{m}^{2}= & (R+l)^{2} \dot{\theta}^{2}+2(R+l)(\dot{y} \cos \phi-\dot{x} \sin \phi) \dot{\theta} \cos \theta \\
& +[\dot{x}-(R+l) \dot{\phi} \sin \theta \cos \phi]^{2}+[\dot{y}-(R+l) \dot{\phi} \sin \theta \sin \phi]^{2}, \\
v_{\mu}^{2}= & (R+r)^{2} \dot{\theta}^{2}+\rho^{2}(\dot{\varkappa}-\dot{\theta})^{2}+2 \rho(R+r)(\dot{\varkappa}-\dot{\theta}) \dot{\theta} \cos \varkappa \\
& +2[(R+r) \dot{\theta} \cos \theta+\rho(\dot{\varkappa}-\dot{\theta}) \cos (\varkappa-\theta)][\dot{y} \cos \phi-\dot{x} \sin \phi] \\
& +[\dot{x}-\dot{\phi} \cos \phi((R+r) \sin \theta+\rho \sin (\varkappa-\theta))]^{2} \\
& +[\dot{y}-\dot{\phi} \sin \phi((R+r) \sin \theta+\rho \sin (\varkappa-\theta))]^{2},
\end{aligned}
$$


and

$$
U=M g R \cos \theta+m g(R+l) \cos \theta+\mu g[(R+r) \cos \theta-\rho \cos (\varkappa-\theta)] .
$$

Constraints. The constraints are given by the standard conditions of rolling without slipping:

$$
\dot{x}=-\dot{\psi} R \cos \phi, \quad \dot{y}=-\dot{\psi} R \sin \phi .
$$

Lagrange-d'Alembert Equations. The equations of motion with a control torque $u$ on the pendulum are those derived in the standard way from the Lagrange-d'Alembert principle (see, for example, Bloch, Krishnaprasad, Marsden, and Murray [1996] and references therein):

$$
\begin{aligned}
\frac{d}{d t} \frac{\partial L_{c}}{\partial \dot{\theta}} & =\frac{\partial L_{c}}{\partial \theta}, \\
\frac{d}{d t} \frac{\partial L_{c}}{\partial \dot{\varkappa}} & =\frac{\partial L_{c}}{\partial \varkappa}+u, \\
\frac{d}{d t} \frac{\partial L_{c}}{\partial \dot{\phi}} & =\mathcal{A} \cos \theta \dot{\theta} \dot{\psi}+\mathcal{B} \cos (\varkappa-\theta)(\dot{\varkappa}-\dot{\theta}) \dot{\psi}, \\
\frac{d}{d t} \frac{\partial L_{c}}{\partial \dot{\psi}} & =-\mathcal{A} \cos \theta \dot{\theta} \dot{\phi}-\mathcal{B} \cos (\varkappa-\theta)(\dot{\varkappa}-\dot{\theta}) \dot{\phi},
\end{aligned}
$$

where $L_{c}(\theta, \varkappa, \dot{\theta}, \dot{\varkappa}, \dot{\psi}, \dot{\phi})=L(\theta, \varkappa, \phi, \dot{\theta}, \dot{\varkappa}, \dot{\psi}, \dot{\phi},-\dot{\psi} R \cos \phi,-\dot{\psi} R \sin \phi)$ is the reduced Lagrangian, and

$$
\mathcal{A}=M R^{2}+m R(R+l)+\mu R(R+r), \quad \mathcal{B}=\mu R \rho .
$$

These equations are supplemented, of course, with the constraints so that one has a well posed initial value problem.

Nonholonomic Momenta and Routhian. Following Bloch, Krishnaprasad, Marsden, and Murray [1996] and Zenkov, Bloch, and Marsden [1998], we introduce the nonholonomic momentum and the constrained Routhian by

$$
p_{1}=\frac{\partial L_{c}}{\partial \dot{\phi}}=I_{11} \dot{\phi}+I_{12} \dot{\psi}, \quad p_{2}=\frac{\partial L_{c}}{\partial \dot{\psi}}=I_{21} \dot{\phi}+I_{22} \dot{\psi}
$$

and

$$
\mathcal{R}=\frac{1}{2}\left(g_{11} \dot{\theta}^{2}+2 g_{12} \dot{\theta} \dot{\varkappa}+g_{22} \dot{\varkappa}^{2}\right)-\frac{1}{2} I^{a b} p_{a} p_{b}-U(\theta, \varkappa),
$$


respectively. Here $I^{a b}$ are the components of the inverse inertia tensor, and $g_{i j}$ represents the internal kinetic energy metric. In the setting here the quantities $g_{i j}$ and the inertia tensor components $I_{a b}$ are given by the formulae

$$
\begin{aligned}
g_{11}= & M R^{2}+m(R+l)^{2}+\mu\left[(R+r)^{2}-2(R+r) \rho \cos \varkappa+\rho^{2}\right]+A, \\
g_{12}= & \mu\left[(R+r) \rho \cos \varkappa-\rho^{2}\right], \\
g_{22}= & \mu \rho^{2}, \\
I_{11}= & M R^{2} \sin ^{2} \theta+m(R+l)^{2} \sin ^{2} \theta \\
& +\mu[(R+r) \sin \theta+\rho \sin (\varkappa-\theta)]^{2}+A \cos ^{2} \theta+B \sin ^{2} \theta, \\
I_{12}= & M R^{2} \sin \theta+m R(R+l) \sin \theta \\
& +\mu R[(R+r) \sin \theta+\rho \sin (\varkappa-\theta)]+B \sin \theta, \\
I_{22}= & M R^{2}+m R^{2}+\mu R^{2}+B .
\end{aligned}
$$

Reduced Equations. Using the symmetry of the system, the variables $(x, y, \phi, \psi)$ can be eliminated by taking the quotient by the action of the group $S O(2) \times S E(2)$. Carrying this out, the resulting reduced equations of motion may be written in terms of the Routhian as

$$
\begin{aligned}
\frac{d}{d t} \frac{\partial \mathcal{R}}{\partial \dot{\theta}} & =\nabla_{\theta} \mathcal{R} \\
\frac{d}{d t} \frac{\partial \mathcal{R}}{\partial \dot{\varkappa}} & =\nabla_{\varkappa} \mathcal{R}+u, \\
\frac{d p_{1}}{d t} & =\left[I^{21} p_{1}+I^{22} p_{2}\right][\mathcal{A} \cos \theta \dot{\theta}+\mathcal{B} \cos (\varkappa-\theta)(\dot{\varkappa}-\dot{\theta})], \\
\frac{d p_{2}}{d t} & =-\left[I^{11} p_{1}+I^{12} p_{2}\right][\mathcal{A} \cos \theta \dot{\theta}+\mathcal{B} \cos (\varkappa-\theta)(\dot{\varkappa}-\dot{\theta})] .
\end{aligned}
$$

The first two of these equations describe the tilting motion of the diskpendulum system, while the second two model the (coupled) wheel dynamics. The covariant derivatives in equations (2.1) and (2.2) are defined by

$$
\begin{aligned}
\nabla_{\theta}=\frac{\partial}{\partial \theta}+[\mathcal{A} \cos \theta- & \mathcal{B} \cos (\varkappa-\theta)] \\
\times & {\left[\left(I^{21} p_{1}+I^{22} p_{2}\right) \frac{\partial}{\partial p_{1}}-\left(I^{11} p_{1}+I^{12} p_{2}\right) \frac{\partial}{\partial p_{2}}\right], } \\
\nabla_{\varkappa}=\frac{\partial}{\partial \varkappa}+\mathcal{B} \cos (\varkappa-\theta) & {\left[\left(I^{21} p_{1}+I^{22} p_{2}\right) \frac{\partial}{\partial p_{1}}-\left(I^{11} p_{1}+I^{12} p_{2}\right) \frac{\partial}{\partial p_{2}}\right] . }
\end{aligned}
$$


The full dynamics is governed by equations (2.1)-(2.4) coupled with the reconstruction equation for the group variables $(x, y, \phi, \psi)$. This reconstruction equation is not needed here as it does not affect the evolution of the shape variables $(\theta, \varkappa)$ describing the internal configuration of the system, and the momentum variables $\left(p_{1}, p_{2}\right)$. The reconstruction equation is thus not used in our stabilization analysis. This is because our stabilization is done modulo the group action, which is natural for the problem. See Zenkov, Bloch, and Marsden [1998] for additional information about the formalism we are using here.

\section{Feedback Stabilization}

In this section we provide a feedback law for the control torque $u$ that stabilizes slow vertical steady state motions of the unicycle with rider moving along a straight line. To put this in context, it is useful to recall that fast steady state motions of the unicycle without rider are already stable, so do not require stabilization (Zenkov, Bloch, and Marsden [1998]). It is this fact that makes fast motions of the unicycle with rider easier to stabilize than slow motions.

We introduce a single control into the pendulum. One can think of this as a controlled limb of the rider. Of course one can introduce a forward motion or steering control for the unicycle, but this is not key to the stability analysis here. Such controls for the wheel are discussed for example in Bloch, Reyhanoglu, and McClamroch [1992], Bloch, Krishnaprasad, Murray, and Marsden [1996] and references therein.

Lyapunov-Malkin Theorem. Our stability analysis is based on the following theorem (see Lyapunov [1992] and Malkin [1938]):

Theorem 3.1 (Lyapunov-Malkin) Consider the system of differential equations

$$
\dot{r}=A r+R(r, s), \quad \dot{s}=S(r, s),
$$

where $r \in \mathbb{R}^{m}, s \in \mathbb{R}^{n}, A$ is an $m \times m$-matrix, and $R(r, s), S(r, s)$ represent higher order nonlinear terms. If all eigenvalues of the matrix A have negative real parts, and $R(r, s), S(r, s)$ vanish when $r=0$, then the solution $r=0$, $s=0$ of this system is stable with respect to $(r, s)$, and asymptotically stable with respect to $r$. If a solution $(r(t), s(t))$ is close enough to the solution 
$r=0, s=0$, then there is a constant n-vector $c$ (depending on the initial conditions) such that

$$
\lim _{t \rightarrow \infty} r(t)=0, \quad \lim _{t \rightarrow \infty} s(t)=c .
$$

This theorem was used by a number of authors in analyzing stability of nonholonomic systems. See Karapetyan [1981], Markeev [1992], Zenkov, Bloch, and Marsden [1998] and references therein. In particular, we stress that the conditions $R(0, s)=0$ and $S(0, s)=0$ are fulfilled for the systems considered in Bloch, Krishnaprasad, Marsden, and Murray [1996] and in Zenkov, Bloch, and Marsden [1998].

Preliminaries on Transforming the Equations into Lyapunov-Malkin Form. We next make some transformations of variables and substitutions that transform equations (2.1)-(2.4) into equations of the form (3.1). Consider an upright steady state motion of the unicycle along a straight line represented by the relative equilibrium

$$
\theta=0, \quad \varkappa=0, \quad p_{1}=0, \quad p_{2}=p_{2}^{0} .
$$

Define new variables $z=\left(z_{1}, z_{2}\right)$ by a linear substitution

$$
p_{1}=z_{1}+I_{0}^{22} p_{2}^{0}[\mathcal{A} \theta+\mathcal{B}(\varkappa-\theta)], \quad p_{2}=p_{2}^{0}+z_{2} .
$$

In the new coordinates, equilibrium (3.3) becomes

$$
\theta=0, \quad \varkappa=0, \quad z_{1}=0, \quad z_{2}=0 .
$$

Moreover, this substitution eliminates linear terms in equations (2.3) and (2.4). Here and below all tensors and derivatives are evaluated at the relative equilibrium (3.3). Equations (2.1)-(2.4) become

$$
\begin{aligned}
g_{11}^{0} \ddot{\theta}+g_{12}^{0} \ddot{\varkappa} & =\nabla_{\theta} \nabla_{\theta} \mathcal{R} \theta+\nabla_{\varkappa} \nabla_{\theta} \mathcal{R} \varkappa-\partial_{\theta} I^{12} p_{2}^{0} z_{1}+\{\text { nonlinear terms }\}, \\
g_{12}^{0} \ddot{\theta}+g_{22}^{0} \ddot{\varkappa} & =\nabla_{\theta} \nabla_{\varkappa} \mathcal{R} \theta+\nabla_{\varkappa} \nabla_{\varkappa} \mathcal{R} \varkappa-\partial_{\varkappa} I^{12} p_{2}^{0} z_{1}+u+\{\text { nonlinear terms }\}, \\
\dot{z}_{1} & =Z_{1}\left(\vartheta, \varkappa, \dot{\vartheta}, \dot{\varkappa}, z_{1}, z_{2}\right), \\
\dot{z}_{2} & =Z_{2}\left(\vartheta, \varkappa, \dot{\vartheta}, \dot{\varkappa}, z_{1}, z_{2}\right),
\end{aligned}
$$

where $Z_{1}$ and $Z_{2}$ are expressions derived from the momentum equations for $p_{1}$ and $p_{2}$ that have been rewritten in terms of $z_{1}$ and $z_{2}$. We do not require the explicit expressions for $Z_{1}$ and $Z_{2}$, but we do need to note that $Z_{1}$ and $Z_{2}$ vanish when $\dot{\theta}=\dot{\varkappa}=0$. 
Feedback Law. Now we introduce the following form of the linear feedback control

$$
u=k_{1} \theta+k_{2} \varkappa+k_{3} \dot{\theta}+k_{4} \dot{\varkappa} .
$$

The shape equations (the first two of equations (2.1)-(2.4)), after being solved for $\ddot{\theta}$ and $\ddot{\varkappa}$, take the form

$$
\dot{v}=y, \quad \dot{y}=B_{k} v+C_{k} y+D_{k} z+W(v, y, z),
$$

where $v=(\theta, \varkappa), y=(\dot{\theta}, \dot{\varkappa}), B_{k}, C_{k}$ and $D_{k}$ are $k$-dependent matrices, and $W(v, y, z)$ represents the nonlinear terms. Next, we show the existence of a linear control law that forces all eigenvalues of the matrix

$$
\left(\begin{array}{cc}
0 & I \\
B_{k} & C_{k}
\end{array}\right)
$$

to belong to the left half plane. Notice that the coefficients of the characteristic polynomial $\lambda^{4}+a_{1} \lambda^{3}+a_{2} \lambda^{2}+a_{3} \lambda+a_{4}$ of the matrix (3.4) are affine functions in the gain parameters $k=\left(k_{1}, k_{2}, k_{3}, k_{4}\right)$. That is, there is a matrix $L$ and a vector $M$ such that $a=\left(a_{1}, a_{2}, a_{3}, a_{4}\right)$ is given by

$$
a=L k+M \text {. }
$$

Direct computation shows that

$$
\operatorname{det} L=-\left(C\left(p_{2}^{0}\right)^{2}+D\right)^{2}
$$

where

$$
\begin{aligned}
& C=\frac{B[R+r-\rho]\left[M R^{2}+m R(R+l)+\mu R(R+r-\rho)+B\right]}{A \mu \rho^{3}\left[M R^{2}+m(R+l)^{2}+A\right]^{2}\left[(M+m+\mu) R^{2}+B\right]^{2}}, \\
& D=\frac{g[(l+\rho-r)(M R+m R+m l)-M R l+A]}{\mu \rho^{3}\left[M R^{2}+m(R+l)^{2}+A\right]^{2}},
\end{aligned}
$$

from which one can see that $\operatorname{det} L \neq 0$ generically. Using formulae (3.5) and (3.6), one finds that the Routh-Hurwitz conditions

$$
a_{1}>0, \quad a_{1} a_{2}-a_{3}>0, \quad\left(a_{1} a_{2}-a_{3}\right) a_{3}-\left(a_{1}\right)^{2} a_{4}>0, \quad a_{4}>0
$$

for the spectrum of matrix (3.4) to be in the left half plane can be satisfied by an appropriate choice of the gain parameters.

Final Transformation into Lyapunov-Malkin Form. When (3.7) is satisfied, det $B_{k} \neq 0$, and so, by the implicit function theorem, there is a unique solution $v=\chi(z)$ of the equation

$$
B_{k} v+D_{k} z+W(v, 0, z)=0 .
$$


Introduce new variables $x$ by

$$
v=x+\chi(z)
$$

Written in the new variables $(x, y, z)$, equations $(2.1)-(2.4)$ become

$$
\begin{aligned}
& \dot{x}=y+X(x, y, z), \\
& \dot{y}=B_{k} x+C_{k} y+Y(x, y, z), \\
& \dot{z}=Z(x, y, z),
\end{aligned}
$$

where

$$
\begin{aligned}
& X(x, y, z)=-\frac{\partial \chi}{\partial z} Z(x, y, z) \\
& Y(x, y, z)=B_{k} \chi(z)+D_{k} z+W(x+\chi(z), y, z) \\
& Z(x, y, z)=Z(x+\chi(z), y, z) .
\end{aligned}
$$

Observe that the nonlinear terms $X(x, y, z), Y(x, y, z)$, and $Z(x, y, z)$ vanish when $x=0$ and $y=0$, because $Z(x, 0, z) \equiv 0$ and $B_{k} \chi(z)+D_{k} z+$ $W(\chi(z), 0, z) \equiv 0$. The equations of motion are thus transformed into the Lyapunov-Malkin form (3.1) with $r=(x, y)$ and $s=z$.

The Main Result. Applying the Lyapunov-Malkin theorem to the setup just described, we find that the equilibrium (3.3) is stable. Summarizing, we have:

Theorem 3.2 The conditions (3.7) define an open non-empty region $\mathcal{S}$ in the space of gain parameters $k=\left(k_{1}, k_{2}, k_{3}, k_{4}\right)$. This is the stability region for the problem in the sense that for any $\left(k_{1}, k_{2}, k_{3}, k_{4}\right) \in \mathcal{S}$, the spectrum of (3.4) belongs to the left half plane and therefore by the Lyapunov-Malkin theorem the steady state motion (3.3) is stable, and asymptotically stable with respect to coordinates $(x, y)$.

It follows from (3.8) that

$$
(\theta, \varkappa)=x+\left(\gamma_{\theta}, \gamma_{\varkappa}\right) z_{1}+\{\text { nonlinear terms }\}
$$

where $\left(\gamma_{\theta}, \gamma_{\varkappa}\right)$ is given by

$$
\left(\begin{array}{c}
\gamma_{\theta} \\
\gamma_{\varkappa}
\end{array}\right)=p_{2}^{0}\left(\begin{array}{cc}
\nabla_{\theta} \nabla_{\theta} \mathcal{R} & \nabla_{\varkappa} \nabla_{\theta} \mathcal{R} \\
\nabla_{\theta} \nabla_{\varkappa} \mathcal{R}+k_{1} & \nabla_{\varkappa} \nabla_{\varkappa} \mathcal{R}+k_{2}
\end{array}\right)^{-1}\left(\begin{array}{c}
\partial_{\theta} I^{12} \\
\partial_{\varkappa} I^{12}
\end{array}\right)
$$


and all derivatives are evaluated at the relative equilibrium (3.3). According to $(3.2)$, this results in

$$
\theta \rightarrow c_{\theta}, \quad \varkappa \rightarrow c_{\varkappa}, \quad \dot{\theta} \rightarrow 0, \quad \dot{\varkappa} \rightarrow 0 \quad \text { as } \quad t \rightarrow \infty
$$

for the stabilized perturbed motions of the unicycle. This means that the unicycle generically approaches a tilted circular steady state motion. Some simulation results will be presented in the next section. As in Bloch, Reyhanoglu, and McClamroch [1992] for example, one needs a discontinuous feedback controller for the system to be asymptotically stabilized about the straight steady state motion $(\theta=0)$.

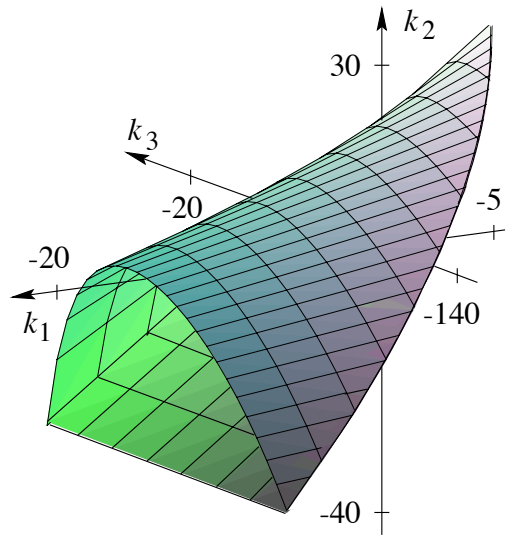

Figure 2: The section of stability region $\mathcal{S}$ for $k_{4}=-100$ and $p_{2}^{0}=6$.

Figure 2 illustrates a typical section of the 4-dimensional stability region $\mathcal{S}$ by a hyperplane $k_{4}=$ const. The stability region is bounded by the surface and the plane shown on this figure. Here and below we choose the following (dimensionless) parameter values: $M=1, m=3, \mu=5, R=1, r=7$, $l=6, \rho=6, A=M R^{2} / 4, B=M R^{2} / 2$, and $g=1$. For slower motions these sections become flat in the $k_{2}$-direction, i.e. the system becomes more $k_{2}$-sensitive.

\section{Simulations}

The following two graphs of $\theta$ as a function of $t$ illustrate the controlled unicycle dynamics. The unicycle starts from

$$
\left(\theta(0), \dot{\theta}(0), \varkappa(0), \dot{\varkappa}(0), p_{1}(0), p_{2}(0)\right)=\left(\theta_{0}, 0,0,0,0,0.1\right) .
$$


For the first graph we choose $\theta_{0}=0.1$ radians and $k_{1}=-300, k_{2}=-200$, $k_{3}=-300$, and $k_{4}=-150$. The eigenvalues of the linearized equations in this case are complex conjugate pairs with negative real parts. The second graph shows that our controller is capable of stabilizing the unicycle from a larger initial displacement $\left(\theta_{0}=0.2\right.$ radians $)$ if the gain parameters are sufficiently large $\left(k_{1}=-3000, k_{2}=-2000, k_{3}=-6000\right.$, and $\left.k_{4}=-3000\right)$. All eigenvalues of the linearization for these values of the gain parameters are real and negative. Nevertheless, we observe a few oscillations before $\theta$ stabilizes. These oscillations reflect the nonlinearity of our coupled system. We remark that in this simulation $c_{\theta}$ is close to zero, but this will not be the case in general (see theorem 3.2).

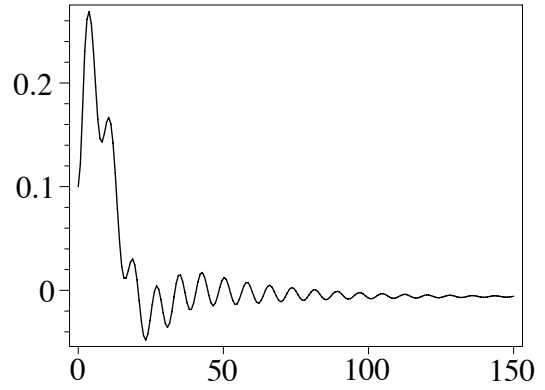

(a) $\theta_{0}=0.1$

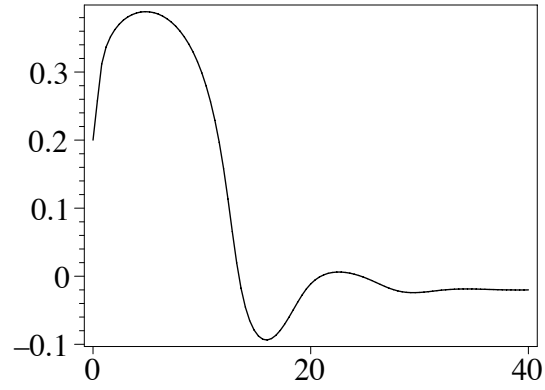

(b) $\theta_{0}=0.2$

Figure 3: Evolution of $\theta$ for various initial conditions.

\section{Conclusions}

This paper has shown that the Lyapunov-Malkin theorem can be used for nonlinear feedback stabilization of a simplified model of the unicycle with a rider. The methodology makes use of a spectral stability condition and leads to a nonlinear stability result. While the system considered here is not feedback linearizable, we expect that the basin of attraction can be expanded by an appropriate choice of nonlinear control terms. We intend to address this issue in a future publication. 


\section{Acknowledgments}

We would like to thank the reviewers for helpful remarks.

\section{References}

Bloch, A.M., P.S. Krishnaprasad, J.E. Marsden, \& R. Murray [1996] Nonholonomic Mechanical Systems with Symmetry. Arch. Rat. Mech. An. 136, 21-99.

Bloch, A.M., N. Leonard, \& J.E. Marsden [1997] Stabilization of Mechanical Systems Using Controlled Lagrangians. Proc. CDC 36, 2356-2361.

Bloch, A.M., N. Leonard, \& J.E. Marsden [1998] Matching and Stabilization by the Method of Controlled Lagrangians. Proc. $C D C$ 37, $1446-1451$.

Bloch, A.M., M. Reyhanoglu, \& N.H. McClamroch [1992] Control and Stabilization of Nonholonomic Dynamic Systems. IEEE Trans. Autom. Contr. 37, 1746-1757.

Karapetyan, A.V. [1981] On Stability of Steady State Motions of a Heavy Solid Body on an Absolutely Rough Horizontal Plane. J. Appl. Math. Mech. 45, 604-608.

Lyapunov, A.M. [1992] The General Problem of the Stability of Motion. London, Washington, DC, Taylor \& Francis.

Malkin, I.G. [1938] On the Stability of Motion in a Sense of Lyapunov (in Russian). Mat. Sbornik XXXV, 47-101.

Markeev, A.P. [1992] Dynamics of a Body Being Contiguous to a Rigid Surface (in Russian). Moscow, Nauka.

Marsden, J.E. [1992] Lectures on Mechanics. London Mathematical Society Lecture Note Series 174, Cambridge University Press.

Zenkov, D.V., A.M. Bloch, \& J.E. Marsden [1998] The Energy-Momentum Method for Stability of Nonholonomic Systems. Dynamics and Stability of Systems 13, 123-165.

Zenkov, D.V., A.M. Bloch, \& J.E. Marsden [1999] Stabilization of the Unicycle with Rider. Proc. CDC 38, 3470-3471. 